\title{
Cisplatin-induced autophagy protects breast cancer cells from apoptosis by regulating yes-associated protein
}

\author{
YULIN JIANG, FEIHU JI, YIFENG LIU, MENGJIA HE, ZHIQIAN ZHANG, JUNHONG YANG, \\ NIAN WANG, CHANGLI ZHONG, QIANNI JIN, XIANGSEN YE and TINGMEI CHEN
}

Key Laboratory of Diagnostic Medicine Designated by the Ministry of Education, Chongqing Medical University, Yuzhong, Chongqing 400016, P.R. China

Received May 29, 2017; Accepted October 5, 2017

DOI: $10.3892 / o r .2017 .6035$

\begin{abstract}
Breast cancer is a common cause of cancer-related deaths in women. Treatment with cisplatin exhibits some therapeutic efficacy. However, treatment optimization is required, and the mechanisms underlying the cisplatin's proapoptotic effects remain unclear. In the present study, we demonstrated that cisplatin induced apoptosis and autophagy in breast cancer cells. Autophagy induced by cisplatin played a protective role in breast cancer cells, which impaired its proapoptotic effect. Mechanistically, for the first time, we found that cisplatin treatment activated the MAPK signaling pathway and promoted autophagy via the ERK signaling pathway. Notably, we found that nuclear translocation of yes-associated protein (YAP) was regulated by cisplatin-induced autophagy, and we identified YAP as a survival input that promoted survival in cisplatintreated breast cancer cells. These findings revealed that administration of cisplatin along with an autophagy inhibitor is a promising therapeutic strategy for treating breast cancer.
\end{abstract}

\section{Introduction}

Breast cancer is the second most common cause of cancerrelated deaths in women (1). It is estimated that $\sim 1.3$ million females develop breast cancer each year, with $\sim 465,000$ expected to succumb to the disease $(2,3)$. Not all patients derive therapeutic benefit from treatment, and some breast cancer patients are resistant to treatment.

Cisplatin, a chemotherapeutic agent, is used for treating various malignancies, including lung, ovary and breast cancer. Mechanistic studies indicated that cisplatin covalently binds to the N-7 atoms of purines on DNA to form DNA adducts, which

Correspondence to: Dr Tingmei Chen, Key Laboratory of Diagnostic Medicine Designated by the Ministry of Education, Chongqing Medical University, 1 Road Yixueyuan, Yuzhong, Chongqing 400016, P.R. China

E-mail: tingmeichen@cqmu.edu.cn

Key words: breast cancer, cisplatin, autophagy, yes-associated protein distort DNA conformation and it also inhibits replication and transcription, leading to cell cycle arrest and apoptosis through the activation of multiple signaling pathways (4). Endoplasmic reticulum (ER) stress is involved in cisplatin-induced apoptosis in human lung cancer cells (5). However, cisplatin exhibits limited therapeutic efficacy (6). Therefore, more effective therapeutic options are required.

There is an established close association between autophagy and cancer, where it has an important role in cell survival and death $(7,8)$. Autophagy is strongly activated by various stress conditions, including cancer chemotherapy and radiotherapy (9). Certain cytotoxic drugs induce protective autophagy, impairing rather than enhancing the action of the drug $(10,11)$. Recent studies have reported that cisplatin treatment activated autophagy, which served as a survival factor to counter cisplatin-induced apoptosis in other cancer cells (12). Mechanically, autophagy activation was tightly regulated by mitogen-activated protein kinases (MAPKs), including extracellular signal-related kinase 1/2 (ERK1/2), p38 and c-jun $\mathrm{N}$-terminal kinase (JNK) (13-15). To the best of our knowledge, there has been no further investigation of the underlying mechanisms between cisplatin and autophagy in breast cancer cells.

The transcriptional co-activator, yes-associated protein (YAP), is an important gene for cell proliferation, metastasis, chemoresistance and other malignant properties (16-18). In breast cancer, the relationship between autophagy and YAP remains controversial. Recent studies, using chemical inhibitors or gene silencing, have reported that autophagy inhibition increased the phosphorylation rate of YAP, promoting the proliferation and invasion of cancer cells $(19,20)$. Meanwhile, YAP enhanced autophagy in response to nutrient deprivation (21). Connecting these phenomena, in the present study, we focused on the communication between YAP and cisplatin-induced autophagy.

\section{Materials and methods}

Cell lines and culture. The human breast cancer (BC) cell lines MCF-7 and MDA-MB-231 cells were obtained from the American Type Culture Collection (ATCC; Rockville, MD, USA). MCF-7 and MDA-MB-231 were cultured in Dulbecco's modified Eagle's medium (DMEM) supplemented with $10 \%$ 
fetal bovine serum (FBS; Gibco, Grand Island, NY, USA), penicillin $(100 \mathrm{U} / \mathrm{ml})$, and streptomycin $(100 \mu \mathrm{g} / \mathrm{ml})$ at $37^{\circ} \mathrm{C}$ with $5 \% \mathrm{CO}_{2}$.

Drugs and reagents. Cisplatin and hydroxychloroquine (HCQ) were purchased from Aladdin (Shanghai, China) and Selleck Chemicals (Houston, TX, USA), respectively. The MAPK inhibitor PD98059 and the JNK inhibitor SP600125 were purchased from Beyotime Biotechnology (Shanghai, China). The p38 inhibitor PD169316 was purchased from MedChem Express (Monmouth Junction, NJ, USA). Hoechst 33342 was purchased from Beijing Solarbio Science \& Technology (Beijing, China). Cisplatin and HCQ were diluted in ultrapure water. Antibodies against $\beta$-actin and YAP were purchased from Santa Cruz Biotechnology, Inc. (Santa Cruz, CA, USA). Antibodies against LC3, p62 and pJNK-Thr183/Tyr185 were purchased from the Novus Biologicals (Littleton, CO, USA), Abcam (Cambridge, MA, USA) and Bioworld Technology (St. Louis Park, MN, USA), respectively. Antibodies against p-YAP, caspase 3, p-p38, p38, pERK-Thr202/Tyr204, ERK and JNK1/2 were purchased from Cell Signaling Technology (Danvers, MA, USA). The horseradish peroxidase (HRP)-conjugated goat anti-mouse, anti-rabbit IgG and FITC-conjugated anti-rabbit IgG secondary antibodies were purchased from Beyotime Biotechnology.

Western blot assay. Human BC cells were lysed in RIPA lysis buffer containing $1 \mathrm{mM}$ phenylmethylsulfonyl fluoride (PMSF) (both from Beyotime Biotechnology) as a protease inhibitor. Protein concentrations were assessed using bicinchoninic acid (BCA) protein assay reagent kit (Beyotime Biotechnology). A total of $40 \mu \mathrm{g}$ of cell lysate was separated by $12 \%$ sodium dodecyl sulfate-polyacrylamide gel electrophoresis (SDS-PAGE), and then transferred onto polyvinylidene difluoride (PVDF) membranes. The membranes were blocked with 5\% non-fat dry milk in Tris-buffered saline with $0.05 \%$ Tween-20 (TBST) for $1 \mathrm{~h}$ followed by incubation at $4^{\circ} \mathrm{C}$ overnight with the primary antibodies. HRP anti-mouse and anti-rabbit $\operatorname{IgG}$ (Beyotime Biotechnology) were used as the secondary antibodies. Immunoreactive bands were detected by ECL chemiluminescent substrate (Thermo Fisher Scientific, Inc., Waltham, MA, USA). The grey value of each band was calculated using ImageJ 1.50i (National Institute of Health, Bethesda, MD, USA) and normalized to the internal control $\beta$-actin.

Hoechst 33342 staining. Human BC cells were fixed for $10 \mathrm{~min}$ in $4 \%$ paraformaldehyde after being treated. The fixed cells were incubated in $0.25 \%$ TritonX-100 for $10 \mathrm{~min}$, then incubated in Hoechst 33342 for 10 min. After being washed three times with phosphate-buffered saline (PBS), the cells were mounted with antifade reagent. Apoptotic cells were identified by the condensation and fragmentation of their nuclei, and were photographed using an Eclipse 80i microscope (Nikon, Tokyo, Japan).

Immunofluorescence microscopy. Human BC cells were fixed for $20 \mathrm{~min}$ in $4 \%$ paraformaldehyde after being treated. The fixed cells were incubated in $0.25 \%$ Triton X-100 for $10 \mathrm{~min}$, and then blocked for $1 \mathrm{~h}$ in $5 \%$ goat serum. The cells were stained with anti-LC3 antibodies (1:50) overnight at $4^{\circ} \mathrm{C}$. After being washed three times with PBS, the cells were stained with appropriate secondary antibodies (1:200) for $1 \mathrm{~h}$ at room temperature. After being washed three times with PBS, the cells were incubated with 4,6-diamidino-2-phenylindole (DAPI) for $10 \mathrm{~min}$ at room temperature. Finally, after being washed another three times with PBS, the cells were mounted with antifade reagent. Fluorescence images were collected using an Eclipse 80i microscope.

Annexin V-FITC/PI double staining. BC cells were detached using EDTA-free trypsin and re-suspended in growth medium. After centrifugation, the cell precipitates were washed with cold PBS three times and resuspended in $1 \mathrm{ml}$ PBS. To determine the rate of apoptosis, the cells were stained with $5 \mu \mathrm{l}$ propidium iodide (PI) and $5 \mu 1$ Annexin V-FITC for $15 \mathrm{~min}$ in the dark. Samples were then analyzed by flow cytometry on a FACScan flow cytometer (BD Biosciences, Franklin Lakes, NJ, USA).

Transmission electron microscopy. For observation under transmission electron microscope (TEM), the cells were first washed with PBS and pelleted by centrifugation before fixing the cell pellet in $2.5 \%$ glutaric dialdehyde and $1 \%$ osmic acid for $2 \mathrm{~h}$. After washing, the pellet was dehydrated and embedded in epoxy resin. Ultrathin sections $(45 \mathrm{~nm})$ were stained with lead acetate, and the stained sections were observed using a transmission electron microscope (H-7500; Hitachi, Tokyo, Japan).

Cell transfection and infection. MCF-7 and MDA-MB-231 cells were plated into 6 -well plates at $2 \times 10^{5}$ cells/well. Lentivirus-based short hairpin RNA (shRNA) vector and lentivirus-based cDNA targeting YAP were transfected into the cells according to the manufacturer's protocol. Cells were selected with puromycin for 10 days at $37^{\circ} \mathrm{C}$. The effectiveness of transfection was ascertained by western blotting. The lentivirus-based shRNA vector and lentivirus-based cDNA targeting YAP were purchased from GenePharma (Shanghai, China). The sequence for YAP shRNA was as follows: YAP, 5'-CCGGGCCACCAAGCTAGATAAAGAACTCGAGTTC TTTATCTAGCTTGGTGGCTTTTTG-3'. The control shRNA was synthesized using a scrambled sequence.

Statistical analysis. Statistical analysis was performed using SPSS for Windows (version 17.0) (SPSS, Inc., Chicago, IL, USA). Student's t-test was used to compare groups. All quantitative results are displayed as the average value \pm the standard deviation. P-values of $<0.05,0.01$ or 0.001 were considered to indicate a statistically significant result.

\section{Results}

Cisplatin induces apoptosis in BC cells. To test the cytotoxic effect of cisplatin on BC cells, the human BC cell lines MCF-7 and MDA-MB-231 were treated with $0,2,4,6,8$ and $10 \mu \mathrm{g} / \mathrm{ml}$ of cisplatin for $48 \mathrm{~h}$. Cell viability was assessed by Cell Counting Kit-8 (CCK-8) cytotoxicity assay. The results revealed that cisplatin-mediated cytotoxicity was dose-dependent, and the half maximal inhibitory concentrations $\left(\mathrm{IC}_{50}\right)$ of cisplatin 
A

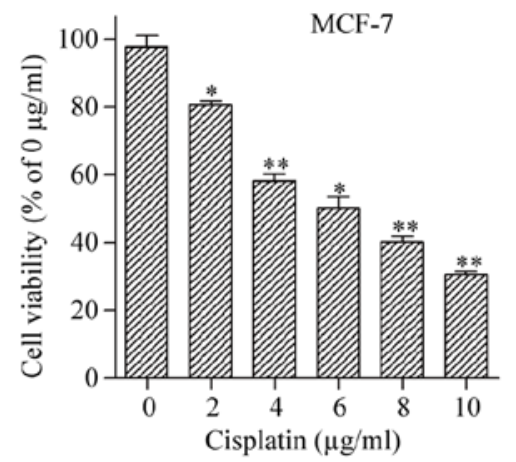

B

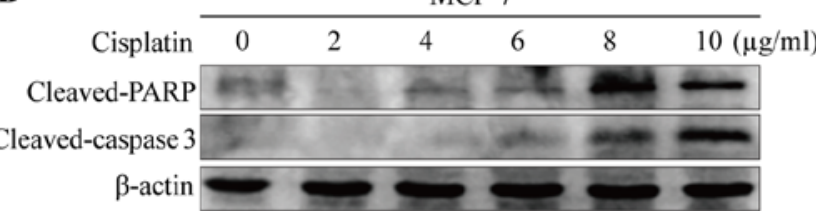

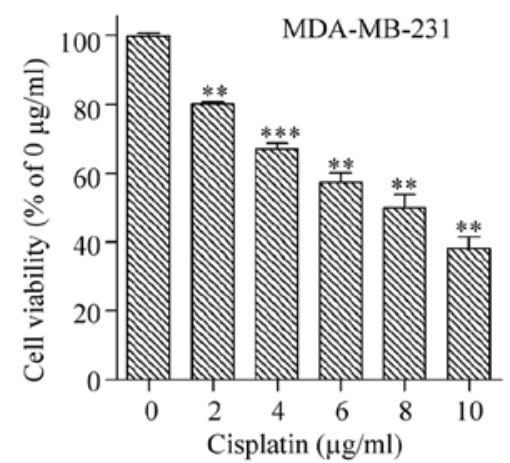

MDA-MB-231

C
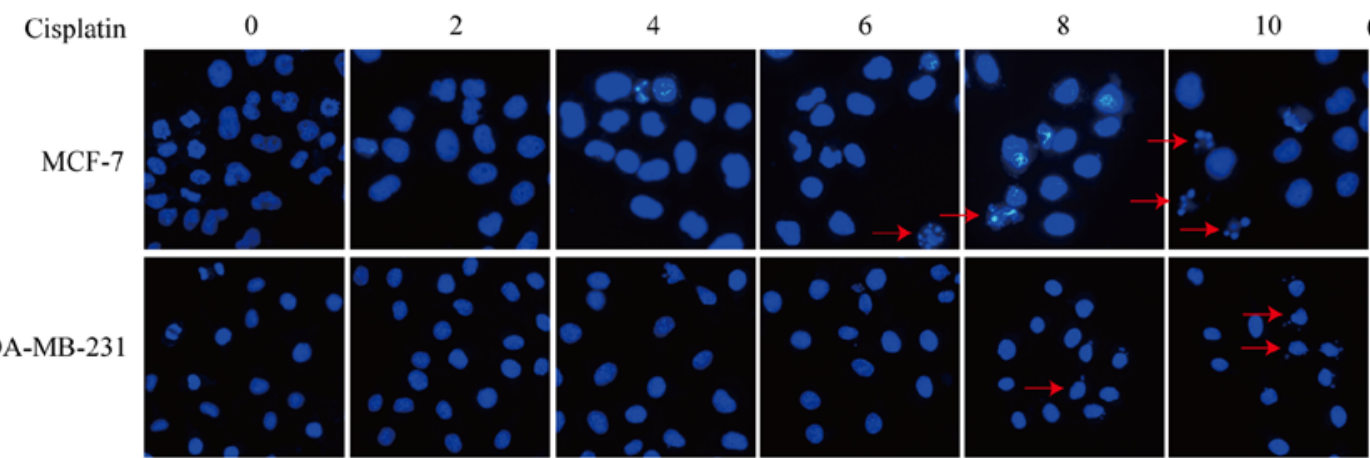

D
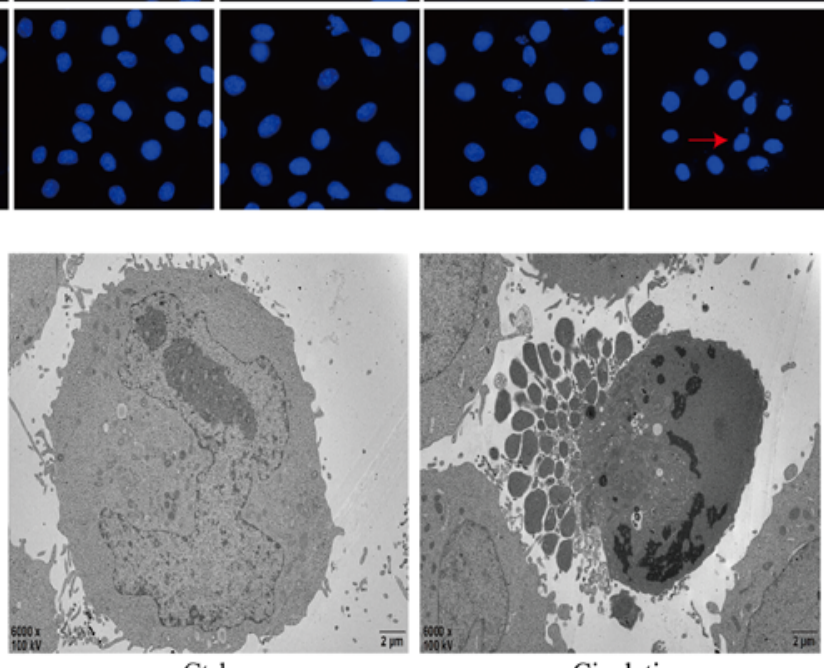

Cisplatin

Figure 1. Cisplatin induces breast cancer cell apoptosis. (A) The cell viability assay examined the cytotoxic effects of cisplatin in MCF-7 and MDA-MB-231 cells for $48 \mathrm{~h}$. (B) Western blotting examined apoptotic markers, cleaved-caspase 3 and cleaved-PARP in MCF-7 and MDA-MB-231 cells with different concentrations of cisplatin treatment. (C) Hoechst 33342 staining revealed apoptotic cells, such as condensed chromatin and nuclear fragmentation indicated by red arrows, after treatment with various concentrations of cisplatin in MCF-7 and MDA-MB-231 cells (original magnification, x400). (D) Transmission electron microscopy was performed to observe typical morphological changes related to apoptosis after cisplatin treatment $(6 \mu \mathrm{g} / \mathrm{ml})$ in MCF-7 cells; " $\mathrm{P}<0.05$, ${ }^{* *} \mathrm{P}<0.01,{ }^{* * *} \mathrm{P}<0.001$. Data are presented as the mean $\pm \mathrm{SD}$ at least three independent experiments.

for MCF-7 and MDA-MB-231 were around 6 and $8 \mu \mathrm{g} /$ $\mathrm{ml}$, respectively (Fig. 1A). Therefore, cisplatin was applied at 6 and $8 \mu \mathrm{g} / \mathrm{ml}$ in subsequent experiments in MCF-7 and MDA-MB-231 cells, respectively. Next, apoptosis was detected by western blot analysis. The levels of cleaved-caspase 3 and cleaved-PARP increased in a dose-dependent manner (Fig. 1B). Compared with the control, the cells exposed to cisplatin had increased dose-dependent apoptosis, with the typical apoptosis morphological changes of condensed nuclear chromatin detected by Hoechst 33342 staining (Fig. 1C). Apoptosis was further evaluated using transmission electron microscopy. We observed cytoplasmic vacuolation, mitochondrion swelling, chromatin condensation, and apoptotic body formation in cisplatin-treated MCF-7 cells (Fig. 1D). These results clearly indicated that cisplatin significantly triggered apoptosis in BC cells.

Cisplatin induces protective autophagy in BC cells. Cisplatin is known to induce autophagy in cancer cells (22). To investigate whether cisplatin induced autophagy in BC cells, we examined autophagy markers LC3 and p62 by immunoblotting. Compared with that of the control, the expression levels of LC3-II increased, and the expression levels of p62 decreased in a dose-dependent manner in MCF-7 and 
A

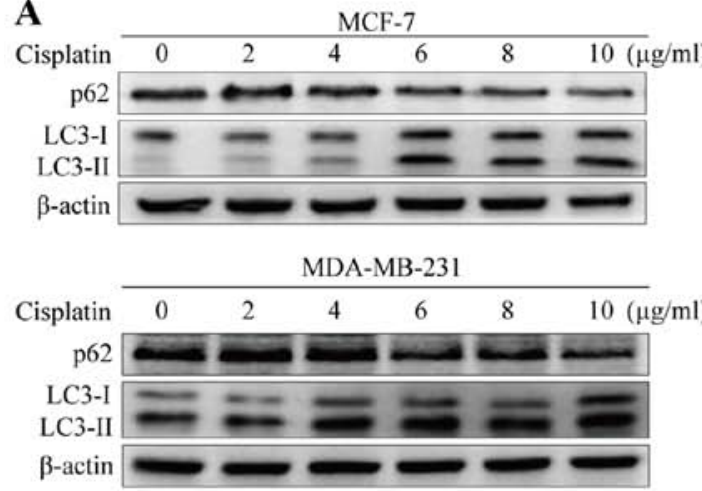

C

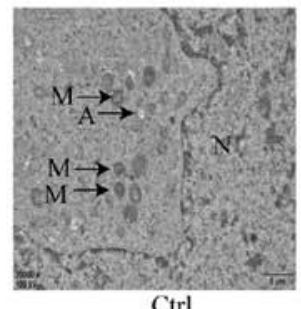

Ctrl

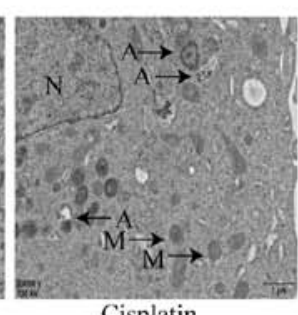

Cisplatin

D

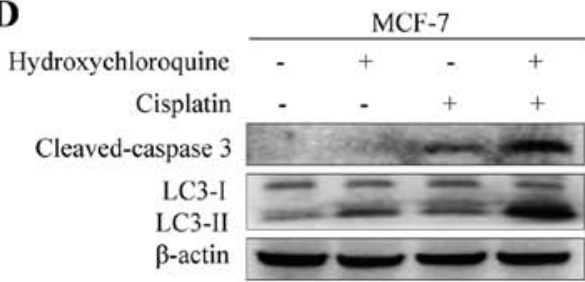

$\mathbf{E}$

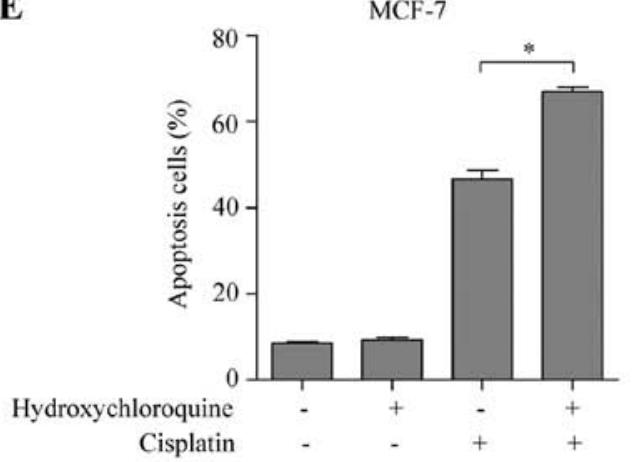

B

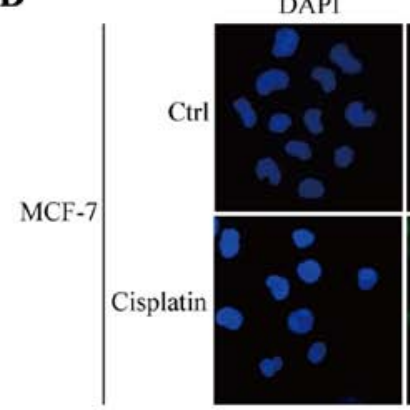

LC3
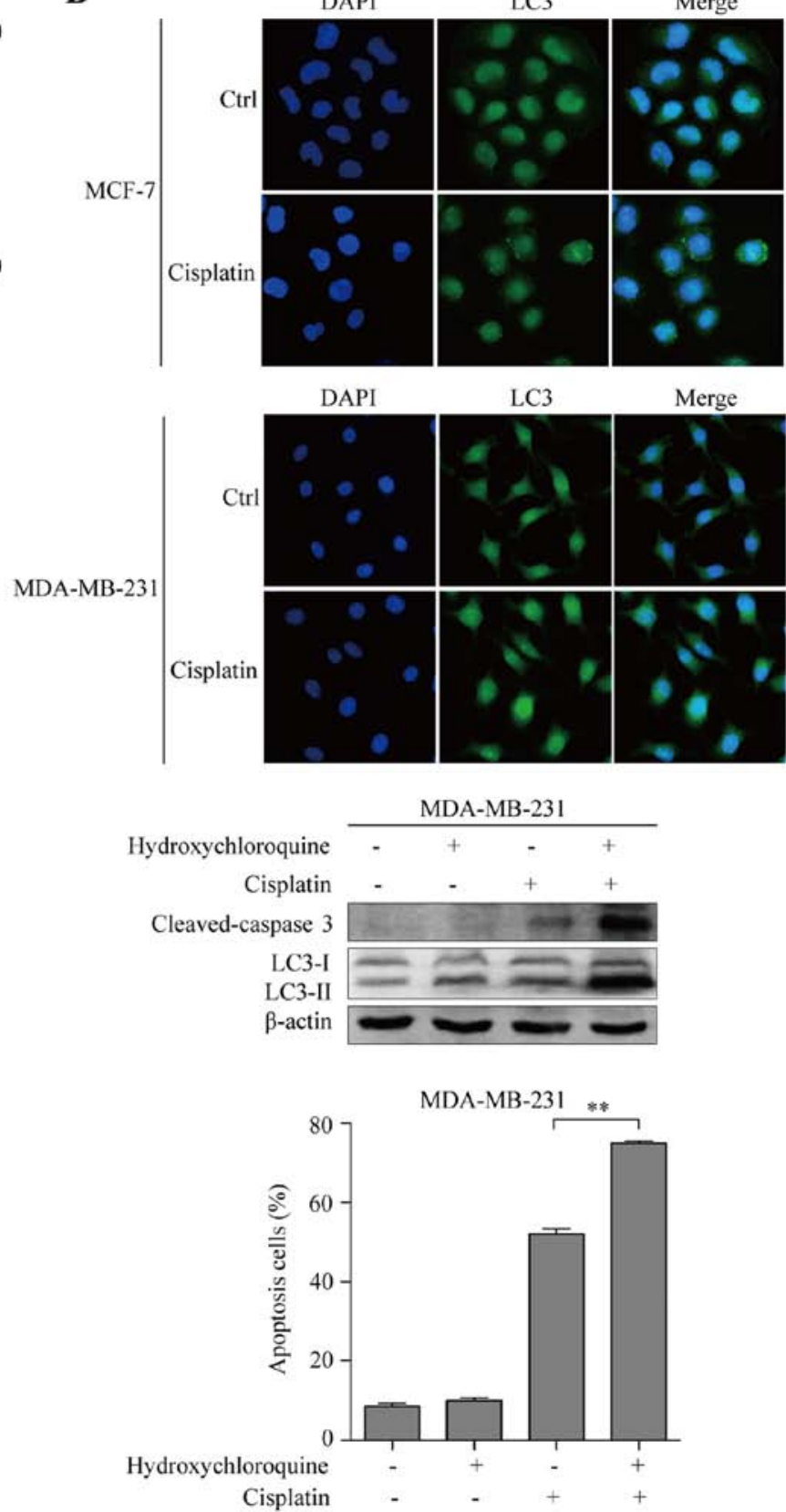

Figure 2. Cisplatin induces protective autophagy in breast cancer cells. (A) Western blotting examined autophagy markers p62/SQSTM1, LC3-I and LC3-II with different concentrations of cisplatin treatment in MCF-7 and MDA-MB-231 cells. (B) Immunfluorescence assay detected LC3 puncta (green) of untreated (control), and cisplatin-treated MCF-7 and MDA-MB-231 cells (original magnification, $\mathrm{x} 400$ ). (C) Transmission electron microscopy was performed to observe autophagosome formation after cisplatin treatment in MCF-7 cells. A, autophagosome; M, mitochondria; N, nucleus. (D) Western blot assay examined apoptotic marker cleaved-caspase 3 of untreated (control), treated with hydroxychloroquine, cisplatin and both these agents in MCF-7 and MDA-MB-231 cells. (E) Quantification of apoptotic cells in untreated (control), treated with autophagy inhibitor hydroxychloroquine, cisplatin and both these agents using Annexin V-FITC/PI double staining in MCF-7 and MDA-MB-231 cells; ${ }^{*} \mathrm{P}<0.05,{ }^{* *} \mathrm{P}<0.01$. Data are presented as the mean \pm SD of at least three independent experiments.

MDA-MB-231 cells after $48 \mathrm{~h}$ of cisplatin treatment (Fig. 2A). To further confirm the role of cisplatin in inducing autophagy, fluorescence microscopy was used to examine the localization of LC3 punctate. Compared with that of the control, we observed increased specific punctate distribution of endogenous LC3 as punctate dots of green fluorescence after $48 \mathrm{~h}$ of cisplatin treatment in both BC cell lines (Fig. 2B). In addition, we examined autophagy using TEM. The control MCF-7 cells had obvious nuclei, and numerous mitochondria. In the cisplatin-treated MCF-7 cells, we observed several clearly visible double-membrane structures resembling autophagosomes, including non-degradable organelles and macromolecules could be observed (Fig. 2C). Collectively, the aforementioned results suggest that cisplatin can induce autophagy in BC cells. To further clarify the function of autophagy as a pro-death or pro-survival mechanism in cisplatin-induced apoptosis, we used the autophagy inhibitor HCQ to suppress the autophagy induced by cisplatin treatment. Immunoblotting assay was used to analyze the activation of cleaved-caspase 3 during the induction of apoptosis. When cells 

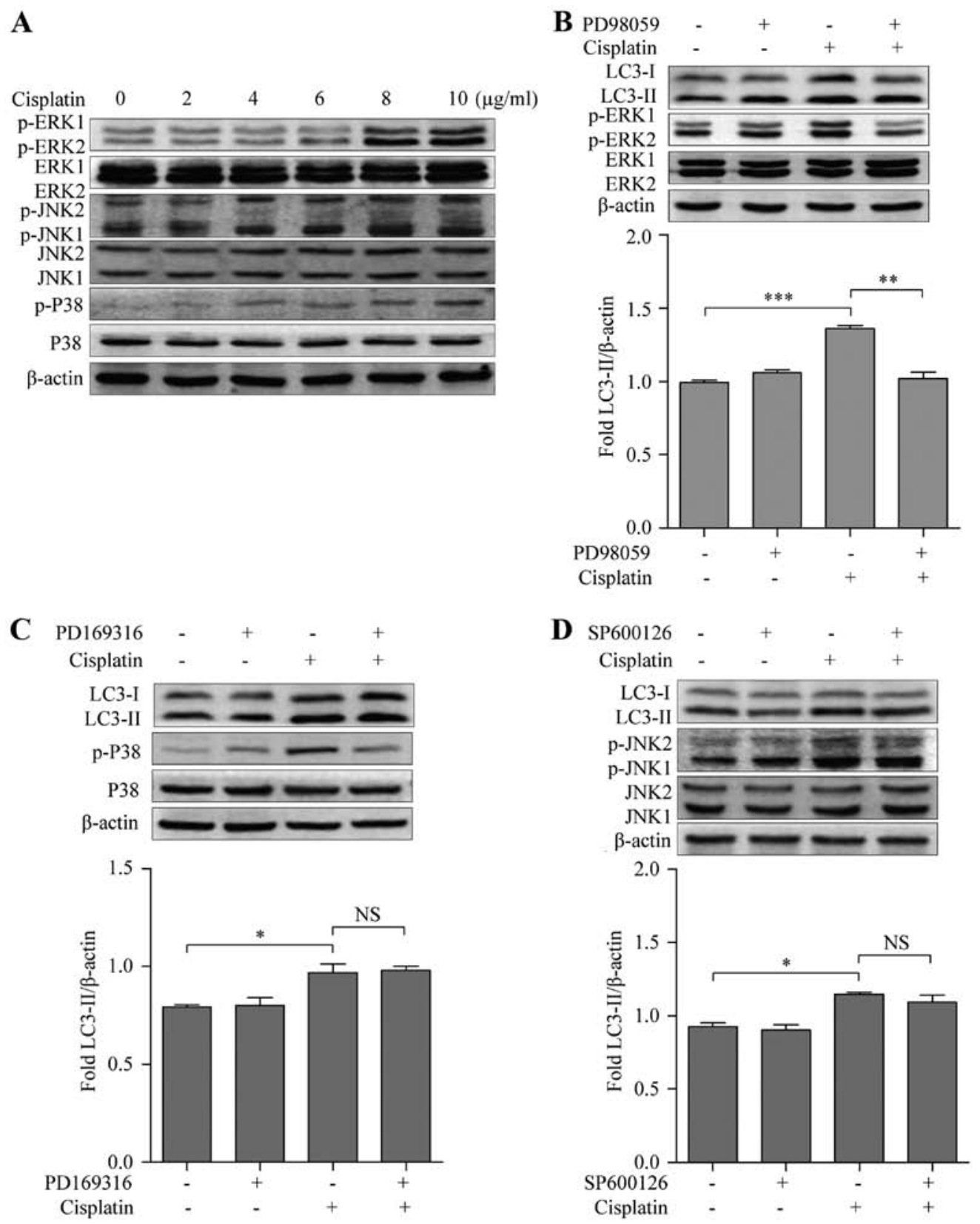

Figure 3. Cisplatin induces autophagy in breast cancer cells via the ERK signaling pathway. (A) Western blotting examined the activation of the MAPK pathways including p-ERK1/2, ERK1/2, p-P38, p38, p-JNK and JNK in MDA-MB-231 cells. (B) Western blot analysis evaluated p-ERK1/2, ERK1/2,LC3 and quantification of LC3-II in untreated (control), treated with the ERK inhibitor PD98059, cisplatin and both these agents in MDA-MB-231 cells. (C) Western blot analysis evaluated p-p38, p38, LC3 and quantification of LC3-II in untreated (control), treated with the p38 inhibitor PD169316, cisplatin, and both these agents in MDA-MB-231 cells. (D) Western blot analysis evaluated p-JNK, JNK, LC3 and quantification of LC3-II in untreated (control), treated with the JNK inhibitor SP600126, cisplatin and both these agents in MDA-MB-231 cells; ${ }^{*} \mathrm{P}<0.05,{ }^{* * *} \mathrm{P}<0.01,{ }^{* * * *} \mathrm{P}<0.001$. Data are presented as the mean \pm SD of at least three independent experiments.

were exposed to cisplatin combined with HCQ, we observed significantly enhanced apoptosis-related changes (Fig. 2D). We then used flow cytometric analysis to confirm the role of autophagy induced by cisplatin treatment in BC cells. Cisplatin combined with HCQ treatment increased the apoptosis rate from 46.8 to $67.0 \%$, and from 52.9 to $74.9 \%$ in MCF-7 and MDA-MB-231 cells, respectively (Fig. 2E). Collectively, these results indicate that inhibition of autophagy enhanced cisplatin-induced apoptosis in BC cells.

Cisplatin promotes autophagy through activation of the ERK signaling pathway. Accumulating evidence indicates that a role for all three MAPK subfamilies in autophagy (23). To investigate whether MAPKs contributed to cisplatininduced autophagy in $\mathrm{BC}$ cells, we assessed the expression levels of phosphorylated ERK1/2, p38 and c-Jun N-terminal kinases (JNK) by immunoblotting. MDA-MB-231 cells were treated with $0,2,4,6,8$ and $10 \mu \mathrm{g} / \mathrm{ml}$ of cisplatin for $48 \mathrm{~h}$, and the levels of p-ERK1/2, p-p38 and p-JNK increased in a dose-dependent manner compared to that of the control, suggesting that cisplatin activated all three MAPK pathways in BC cells (Fig. 3A). To determine which MAPK is involved in cisplatin-induced autophagy, ERK inhibitor PD98059 (10 $\mu \mathrm{mol} / \mathrm{ml})$, p38 inhibitor PD169316 $(10 \mu \mathrm{mol} / \mathrm{ml})$ and JNK 
A

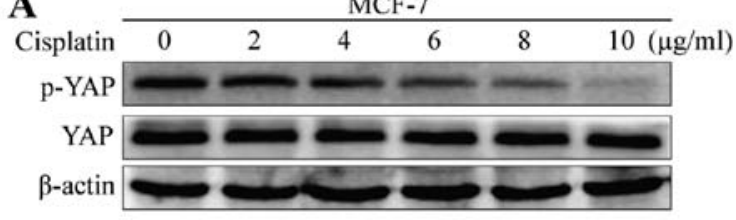

B

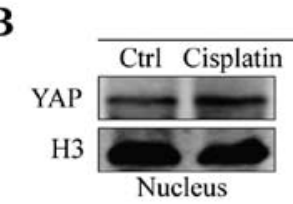

MCF-7

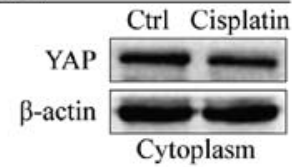

C

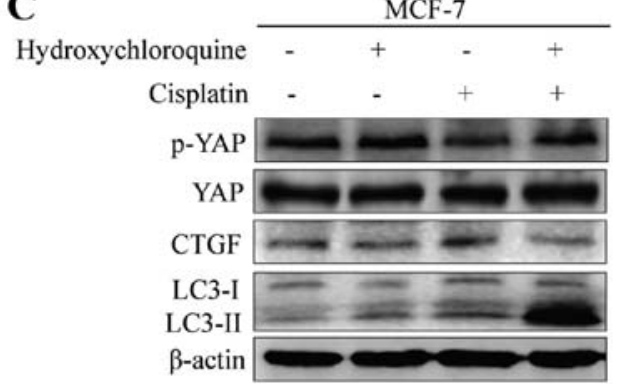

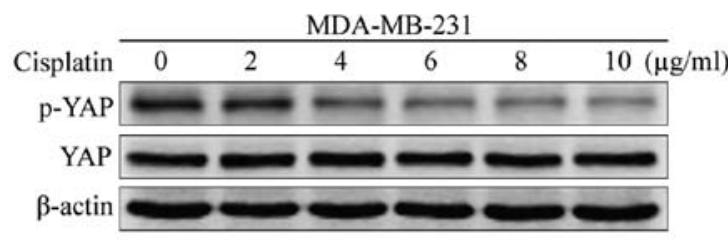
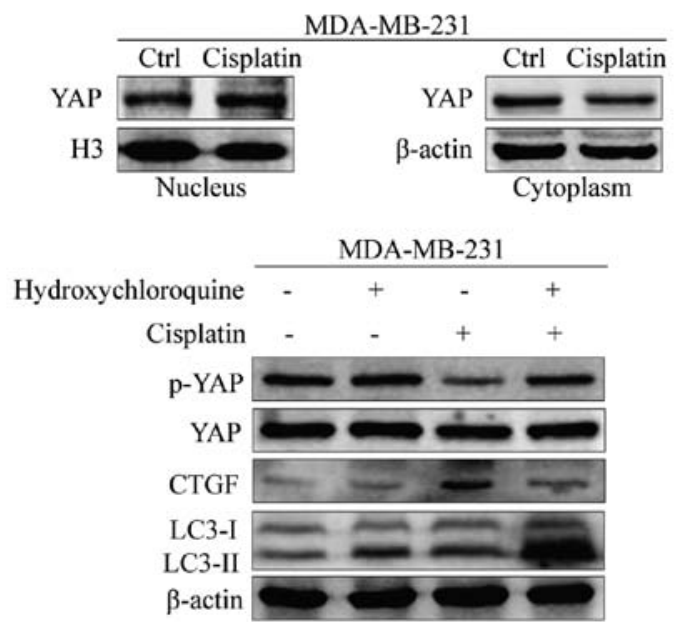

Figure 4. Cisplatin-induced autophagy promotes YAP activity. (A) Western blot assay examined p-YAP, YAP after various concentrations of cisplatin treatment in MCF-7 and MDA-MB-231 cells. (B) Western blot assay evaluated the nuclear and cytoplasmic YAP after cisplatin treatment in MCF-7 and MDA-MB-231 cells. (C) Western blot assay examined p-YAP, YAP, CTGF and LC3 in untreated (control), treated with autophagy inhibitor hydroxychloroquine, cisplatin, and both these agents in MCF-7 and MDA-MB-231 cells. Data are presented as the mean \pm SD of at least three independent experiments.

inhibitor SP600126 (10 $\mu \mathrm{mol} / \mathrm{ml})$ were used. MDA-MB-231 cells exposed to cisplatin combined with the ERK inhibitor, but not the p38 or JNK inhibitors, had decreased LC3-II when compared to cisplatin alone (Fig. 3B-D), suggesting that the ERK signaling pathway may be involved in cisplatin-induced autophagy.

Cisplatin-induced autophagy promotes YAP activity. The transcription co-activator YAP is thought to be involved in autophagy-dependent proliferation and invasion of BC cells (19). However, the role of YAP activation under cisplatin-induced autophagy remains unknown. The rate of phosphorylated YAP decreased with cisplatin treatment in a dose-dependent manner in MCF-7 and MDA-MB-231 cells (Fig. 4A). Consistent with this observation, immunoblotting of the nuclear and cytoplasmic fractions revealed increased accumulation of YAP protein in the nucleus of MCF-7 and MDA-MB-231 cells with cisplatin treatment (Fig. 4B). As YAP could be regulated by autophagy, we validated the impact of autophagy on YAP phosphorylation using the autophagy inhibitor HCQ. Compared with cisplatin alone, phosphorylation of YAP increased when treated with cisplatin and HCQ in MCF-7 and MDA-MB-231 cells (Fig. 4C). In addition, we found that cisplatin-induced autophagy increased the expression of CTGF, the target gene of YAP. These results indicate that cisplatin-induced autophagy had a significant impact on YAP activity.

Transcription co-activator YAP contributes to cisplatin-induced apoptosis. Since cisplatin-induced autophagy has a significant impact on YAP activity, we next investigated the role of YAP in the apoptosis of cisplatin-treated cells. MCF-7 and MDA-MB-231 cells were transfected with either non-target (shNC) or YAP shRNAs (shYAP), and the expression of YAP was examined by western blotting. Compared with the control-transfected cells, the amount of YAP in cells transfected with YAP shRNA decreased significantly (Fig. 5A). Next, we used a CCK-8 assay to examine the cytotoxic effects of shNC and shYAP. Depletion of YAP significantly increased the cytotoxicity of cisplatin in MCF-7 and MDA-MB-231 cells (Fig. 5B). Consistent with this observation, we also found that the apoptotic marker cleaved-caspase 3 was increased in YAP knockdown cisplatin-treated cells, compared with that of their control shRNA-expressing counterparts (Fig. 5C). This data indicated that YAP inactivation was essential for the apoptotic effect of cisplatin in BC cells. Collectively, these results revealed that cisplatin induced autophagy, which functioned as a protective mechanism by regulating nuclear translocation of YAP, and subsequently impairing the apoptosis of BC cells (Fig. 5D).

\section{Discussion}

Cisplatin is a chemotherapeutic agent used for treating various malignancies, and functions by causing lethal DNA damage and inducing apoptosis (24). Although there are obvious benefits of cisplatin-based treatment of breast cancer, accumulating evidence indicates that the efficacy of cisplatin is still a problem (25). Multiple mechanisms contribute to the efficacy of cisplatin treatment in cancer cells. For example, changes in cytokine and phosphorylation profiles of human mesenchymal stromal cells led to increased chemoresistance and stemness of breast cancer cells. MicroRNA-302b overexpression enhanced cisplatin sensibility of breast cancer cells by inducing apoptosis, but the mechanisms underlying this 

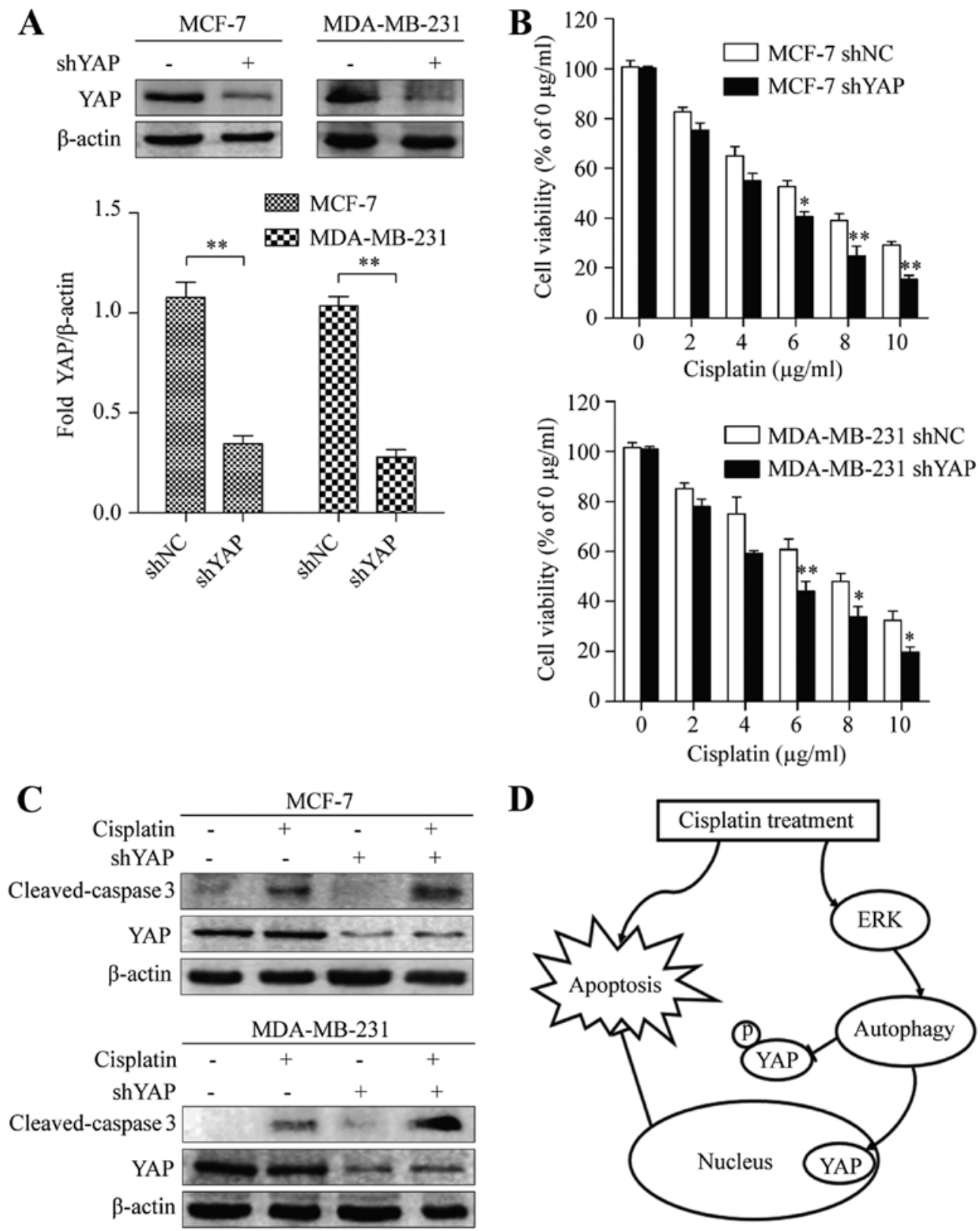

Figure 5. YAP regulates the cytotoxic effect of cisplatin in breast cancer cells. (A) Western blot assay examined YAP and quantification of YAP after YAP knockdown in MCF-7 and MDA-MB-231 cells. (B) CCK-8 assay evaluated the cytotoxic effects of cisplatin after YAP knockdown in MCF-7 and MDAMB-231 cells. (C) Western blot assay examined cleaved-caspase 3 of cisplatin treatment after YAP knockdown in MCF-7 and MDA-MB-231 cells. (D) The schematic model for the mechanism by which cisplatin activates autophagy via the ERK signaling pathway, which subsequently promotes the nuclear location of YAP, reducing the cytotoxic effect of cisplatin in breast cancer cells; ${ }^{*} \mathrm{P}<0.05,{ }^{* * *} \mathrm{P}<0.01$. Data are presented as the mean $\pm \mathrm{SD}$ of at least three independent experiments.

effect are not fully understood (26). Investigation of cisplatin signal transduction is of particular importance. Recent studies suggest that the cytotoxic effects of drugs could by mediated by autophagy (27).

Cisplatin has been reported to promote apoptosis and inhibit proliferation in breast cancer cells (28). Consistently, in the present study we demonstrated that cisplatin exhibited significant cytotoxic effects in a dose-dependent manner in MCF-7 and MDA-MB-231 cells. Furthermore, we found that cisplatin induced autophagy, which was confirmed by evaluating the expression of several autophagy markers and by electron microscopy. Autophagy plays an important role in cancer treatment $(29,30)$. Certain cytotoxic drugs can induce protective autophagy in cancer cells, impairing the cytotoxic effect of these drugs (31). We speculated that the activation of autophagy weakens cisplatin-induced apoptotic cell death. We used the lysosomal inhibitor hydroxychloroquine (HCQ) to further investigate the function of autophagy in cisplatin treatment. Inhibition of autophagy enhanced cisplatin-induced apoptosis in both MCF-7 and MDA-MB-231 cells, indicated that suppression of autophagy may be a promising strategy for breast cancer therapy using cisplatin.

The mechanisms by which autophagy is regulated are not fully understood. Emerging evidence has revealed that all three MAPK subfamilies regulated autophagy in other cancer cells $(15,32,33)$. For example, a recent study stated that ERK 
promoted autophagy in human melanoma cells by CREB protein (34). The p38 MAPK blockade induced an increase in autophagy through enhanced interactions between p38 interacting protein (p38IP) and autophagy protein 9 (ATG9) in an mTOR-independent manner (35). JNK1-mediated multisite phosphorylation of Bcl-2 stimulated starvation-induced autophagy by disrupting the Bcl-2/Beclin 1 complex (32). In the present study, we found that cisplatin treatment activated the MAPK signaling pathway in breast cancer cells. We used an ERK, p38, and JNK inhibitors to clarify the function of all three MAPKs in the regulation of cisplatin-induced autophagy. We revealed that the p38 and JNK inhibitors did not block cisplatin-induced autophagy. Therefore, we concluded that cisplatin treatment activated autophagy via the ERK signaling pathway.

The transcription co-activator YAP is an important regulator in cancer treatment (36). Notably, the phosphorylation level of YAP decreased with cisplatin treatment, which was accompanied by significant nuclear accumulation of YAP. The upstream kinase molecule LATS was not altered (data not shown), indicating that YAP was regulated by another process. A recent study revealed that YAP was modulated by autophagy, and could control cell proliferation and invasion (19). Consistent with the present study, we found that YAP and the YAP-target gene CTGF were regulated by cisplatininduced autophagy using HCQ. Moreover, YAP depletion potentiated the cytotoxic effects of cisplatin in breast cancer cells. These observations strongly indicated that cisplatininduced autophagy promoted YAP activity, further inhibiting apoptosis with cisplatin treatment in breast cancer cells.

In conclusion, in the present study we demonstrated that the inhibition of autophagy markedly enhanced cisplatin-induced apoptosis in breast cancer cells, and that the transcription co-activator YAP plays an important role in this process. Therefore, the combination of cisplatin and an autophagy inhibitor may provide an effective chemical therapy for breast cancer treatment.

\section{Acknowledgements}

The present study was supported by a grant from the National Natural Science Foundation of China (no. 81272544) to T.C.

\section{References}

1. Dey N, De P and Leyland-Jones B: PI3K-AKT-mTOR inhibitors in breast cancers: From tumor cell signaling to clinical trials. Pharmacol Ther 175: 91-106, 2017.

2. Herranz M and Ruibal A: Optical imaging in breast cancer diagnosis: The next evolution. J Oncol 2012: 863747, 2012.

3. Shah NR and Chen H: MicroRNAs in pathogenesis of breast cancer: Implications in diagnosis and treatment. World J Clin Oncol 5: 48-60, 2014.

4. Miller RP, Tadagavadi RK, Ramesh G and Reeves WB: Mechanisms of cisplatin nephrotoxicity. Toxins 2: 2490-2518, 2010.

5. Shi S, Tan P, Yan B, Gao R, Zhao J, Wang J, Guo J, Li N and Ma Z: ER stress and autophagy are involved in the apoptosis induced by cisplatin in human lung cancer cells. Oncol Rep 35: 2606-2614, 2016.

6. Czarnomysy R, Surażyński A, Popławska B, Rysiak E, Pawłowska N, Czajkowska A, Bielawski K and Bielawska A Synergistic action of cisplatin and echistatin in MDA-MB-231 breast cancer cells. Mol Cell Biochem 427: 13-22, 2017.
7. Gewirtz DA: Cytoprotective and nonprotective autophagy in cancer therapy. Autophagy 9: 1263-1265, 2013.

8. Mah LY and Ryan KM: Autophagy and cancer. Cold Spring Harb Perspect Biol 4: a008821, 2012.

9. Liang DH, El-Zein R and Dave B: Autophagy inhibition to increase radiosensitization in breast cancer. J Nucl Med Radiat Ther 6: 254, 2015.

10. Aydinlik S, Erkisa M, Cevatemre B, Sarimahmut M, Dere E, Ari F and Ulukaya E: Enhanced cytotoxic activity of doxorubicin through the inhibition of autophagy in triple negative breast cancer cell line. Biochim Biophys Acta 1861: 49-57, 2017.

11. Wen J, Yeo S, Wang C, Chen S, Sun S, Haas MA, Tu W, Jin F and Guan JL: Autophagy inhibition re-sensitizes pulse stimulation-selected paclitaxel-resistant triple negative breast cancer cells to chemotherapy-induced apoptosis. Breast Cancer Res Treat 149: 619-629, 2015.

12. Yu L, Gu C, Zhong D, Shi L, Kong Y, Zhou Z and Liu S: Induction of autophagy counteracts the anticancer effect of cisplatin in human esophageal cancer cells with acquired drug resistance. Cancer Lett 355: 34-45, 2014.

13. Basu S, Rajakaruna S, Reyes B, Van Bockstaele E and Menko AS: Suppression of MAPK/JNK-MTORC1 signaling leads to premature loss of organelles and nuclei by autophagy during terminal differentiation of lens fiber cells. Autophagy 10: 1193-1211, 2014.

14. Zeng Y, Yang X, Wang J, Fan J, Kong Q and Yu X: Aristolochic acid I induced autophagy extenuates cell apoptosis via ERK 1/2 pathway in renal tubular epithelial cells. PLoS One 7: e30312, 2012.

15. Yang X, Wang J, Dai J, Shao J, Ma J, Chen C, Ma S, He Q, Luo P and Yang B: Autophagy protects against dasatinib-induced hepatotoxicity via p38 signaling. Oncotarget 6: 6203-6217, 2015.

16. Zhi X, Zhao D, Zhou Z, Liu R and Chen C: YAP promotes breast cell proliferation and survival partially through stabilizing the KLF5 transcription factor. Am J Pathol 180: 2452-2461, 2012.

17. Sorrentino G, Ruggeri N, Zannini A, Ingallina E, Bertolio R, Marotta C, Neri C, Cappuzzello E, Forcato M, Rosato A, et al: Glucocorticoid receptor signalling activates YAP in breast cancer. Nat Commun 8: 14073, 2017.

18. Lamar JM, Stern P, Liu H, Schindler JW, Jiang ZG and Hynes RO: The Hippo pathway target, YAP, promotes metastasis through its TEAD-interaction domain. Proc Natl Acad Sci USA 109: E2441-E2450, 2012.

19. Lefort S, Joffre C, Kieffer Y, Givel AM, Bourachot B, Zago G, Bieche I, Dubois T, Meseure D, Vincent-Salomon A, et al: Inhibition of autophagy as a new means of improving chemotherapy efficiency in high-LC3B triple-negative breast cancers. Autophagy 10: 2122-2142, 2014.

20. Kwon Y, Vinayagam A, Sun X, Dephoure N, Gygi SP, Hong P and Perrimon N: The Hippo signaling pathway interactome. Science 342: 737-740, 2013.

21. Song Q, Mao B, Cheng J, Gao Y, Jiang K, Chen J, Yuan Z and Meng S: YAP enhances autophagic flux to promote breast cancer cell survival in response to nutrient deprivation. PLoS One 10: e0120790, 2015.

22. He J, Yu JJ, Xu Q, Wang L, Zheng JZ, Liu LZ and Jiang BH: Downregulation of ATG14 by EGR1-MIR152 sensitizes ovarian cancer cells to cisplatin-induced apoptosis by inhibiting cytoprotective autophagy. Autophagy 11: 373-384, 2015.

23. Sui X, Kong N, Ye L, Han W, Zhou J, Zhang Q, He C and Pan H: p38 and JNK MAPK pathways control the balance of apoptosis and autophagy in response to chemotherapeutic agents. Cancer Lett 344: 174-179, 2014.

24. Siddik ZH: Cisplatin: Mode of cytotoxic action and molecular basis of resistance. Oncogene 22: 7265-7279, 2003.

25. Skolekova S, Matuskova M, Bohac M, Toro L, Durinikova E, Tyciakova S, Demkova L, Gursky J and Kucerova L: Cisplatin-induced mesenchymal stromal cells-mediated mechanism contributing to decreased antitumor effect in breast cancer cells. Cell Commun Signal 14: 4, 2016.

26. Cataldo A, Cheung DG, Balsari A, Tagliabue E, Coppola V, Iorio MV, Palmieri D and Croce CM: miR-302b enhances breast cancer cell sensitivity to cisplatin by regulating E2F1 and the cellular DNA damage response. Oncotarget 7: 786-797, 2016.

27. Carew JS, Nawrocki ST and Cleveland JL: Modulating autophagy for therapeutic benefit. Autophagy 3: 464-467, 2007.

28. Liang S, Peng X, Li X, Yang P, Xie L, Li Y, Du C and Zhang G: Silencing of CXCR4 sensitizes triple-negative breast cancer cells to cisplatin. Oncotarget 6: 1020-1030, 2015. 
29. Ambjørn M, Ejlerskov P, Liu Y, Lees M, Jäättelä $M$ and Issazadeh-Navikas S: IFNB1/interferon- $\beta$-induced autophagy in MCF-7 breast cancer cells counteracts its proapoptotic function. Autophagy 9: 287-302, 2013.

30. Sui X, Chen R, Wang Z, Huang Z, Kong N, Zhang M, Han W, Lou F, Yang J, Zhang Q, et al: Autophagy and chemotherapy resistance: A promising therapeutic target for cancer treatment. Cell Death Dis 4: e838, 2013

31. Hanahan D and Weinberg RA: Hallmarks of cancer: The next generation. Cell 144: 646-674, 2011

32. Wei Y, Pattingre S, Sinha S, Bassik M and Levine B: JNK1mediated phosphorylation of Bcl-2 regulates starvation-induced autophagy. Mol Cell 30: 678-688, 2008.

33. Li D, Hu J, Wang T, Zhang X, Liu L, Wang H, Wu Y, Xu D and Wen F: Silymarin attenuates cigarette smoke extract-induced inflammation via simultaneous inhibition of autophagy and ERK/p38 MAPK pathway in human bronchial epithelial cells. Sci Rep 6: 37751, 2016.
34. Liu YL, Lai F, Wilmott JS, Yan XG, Liu XY, Luan Q, Guo ST, Jiang CC, Tseng HY, Scolyer RA, et al: Noxa upregulation by oncogenic activation of MEK/ERK through CREB promotes autophagy in human melanoma cells. Oncotarget 5: 11237-11251, 2014.

35. Henson SM, Lanna A, Riddell NE, Franzese O, Macaulay R, Griffiths SJ, Puleston DJ, Watson AS, Simon AK, Tooze SA, et al: p38 signaling inhibits mTORC1-independent autophagy in senescent human $\mathrm{CD}^{+} \mathrm{T}$ cells. J Clin Invest 124: 4004-4016, 2014.

36. Ciamporcero E, Shen $\mathrm{H}$, Ramakrishnan S, Yu Ku S, Chintala S, Shen L, Adelaiye R, Miles KM, Ullio C, Pizzimenti S, et al: YAP activation protects urothelial cell carcinoma from treatmentinduced DNA damage. Oncogene 35: 1541-1553, 2016. 